\section{APLICAÇÃO DOS CONCEITOS DE GOVERNANÇA DAS CONTRATAÇÕES NA CADEIA DE SUPRIMENTOS DE UM HOSPITAL PÚBLICO UNIVERSITÁRIO}

\section{APPLICATION OF PROCUREMENT GOVERNANCE TO THE SUPPLY CHAIN AT A PUBLIC UNIVERSITY HOSPITAL}

\author{
Luana Baptista Rodrigues Pires ${ }^{1}$, Luciane Camillo de Magalhães ${ }^{1}$, \\ Simone Dalla Pozza Mahmud ${ }^{1}$
}

\section{RESUMO}

Introdução: O tema da governança corporativa vem ganhando cada vez mais destaque nas Organizações Públicas, principalmente a governança nas aquisições, pois objetivam agregar valor ao negócio com riscos aceitáveis, através da adoção de boas práticas de gestão, ética e transparência e cumprimento da legislação pública.

Métodos: O trabalho a seguir apresenta um estudo para aprimorar os processos internos da área de Planejamento e Compras em um Hospital Público Universitário, de alta complexidade. As fases de planejamento e compras em uma Instituição hospitalar de grande porte são extremamente desafiadoras visto a variabilidade de produtos necessários para o atendimento das demandas assistenciais, e no caso específico, também de pesquisa e ensino. $O$ volume de processos e de aquisições geradas ao longo do exercício são inúmeras e há um grande contingente de pessoas envolvidas, de forma multidisciplinar. Esse trabalho propôs implementar ações concretas que aprimoram a governança das contratações, com o uso de estratégias e ferramentas voltadas à efetividade dos processos de aquisição, na assertividade, controle e monitoramento dos riscos, mantendo os padrões de qualidade e segurança da Organização.

Resultados: Através deste trabalho, obteve-se o aumento de dez pontos percentuais no aproveitamento nas licitações e redução do tempo de tramitação dos processos em de quarenta e um dias.

Conclusão: A adoção de ferramentas de governança nas aquisições públicas traz melhores resultados de processo e eficiência nas compras públicas e ainda promove a transparência e a visibilidade das ações aos fornecedores.

Palavras-chave: Compras públicas; Otimização; Governança

\section{ABSTRACT}

Introduction: The theme of corporate governance has been gaining increasing prominence in Public Organizations, especially governance in procurement, as it aims to add value to the business with acceptable risks, through the adoption of good management practices, ethics and transparency and compliance with public legislation.

Methods: The following work presents a study to improve the internal processes of the Planning and Purchasing area in a Public University Hospital of high complexity. The planning and purchasing phases in a large hospital are extremely challenging due to the variability of products needed to meet the demands of health care, and in this specific case, also of research and teaching. The volume of processes and acquisitions generated throughout the year are numerous and there is a large contingent of people involved, in a multidisciplinary way. This work proposed to implement concrete actions that improve the governance of procurement, with the use of strategies and tools
Clin Biomed Res. 2021;41(4):319-324

1 Hospital de Clinicas de Porto Alegre. Porto Alegre, RS, Brasil.

Autor correspondente

Luciane Camillo de Magalhães Imagalhaes@hcpa.edu.br Hospital de Clinicas de Porto Alegre Rua Ramiro Barcelos, 2350

90035-903, Porto Alegre, RS, Brasil. 
Pires et al.

aimed at the effectiveness of procurement processes, assertiveness, control and monitoring of risks, while maintaining the standards of quality and safety of the Organization.

Results: Through this work, it was obtained the increase of ten percentage points in the utilization in the bids and reduction of the processing time of the processes in forty-one days.

Conclusion: The adoption of governance tools in public procurement brings better process results and efficiency in public purchases and also promotes transparency and visibility of actions to suppliers.

Keywords: Public procurement; Optimization; Governance

\section{INTRODUÇÃO}

A governança pública é definida pelo Decreto $9.203 / 2017$, como o conjunto de mecanismos de liderança, estratégia e controle postos em prática para avaliar, direcionar e monitorar a gestão, com vistas à condução das políticas públicas e à prestação de serviços de interesse da sociedade. Neste sentido, as compras públicas constituem-se em uma das áreas mais sensíveis e importantes da atividade logística que movimenta a Administração Pública. Além de seu valor estratégico, o processo de compras públicas mobiliza e influencia toda a organização e o ciclo socioeconômico, haja vista o poder de compra do Estado 1 .

A administração pública é conhecida pela burocracia e lentidão dos processos de aquisição em razão das diversas etapas impostas pelas legislações e segmentação de tarefas no serviço público. Para a gestão da cadeia de suprimentos de um Hospital de ensino de alta complexidade estas características impõem um importante desafio, pois se faz necessário garantir os insumos para atendimento assistencial no prazo certo, na quantidade correta, com qualidade e preços adequados. Neste sentido, é preciso buscar ferramentas que visem a otimização dos processos para torná-los mais efetivos, ágeis e assertivos. Para atender essa expectativa, aplicouse os conceitos combinados de três ferramentas de gestão de processos e melhoria contínua que foram aplicadas na governança das aquisições, sendo o método Lean, Strategic Sourcing e Gestão de Riscos ${ }^{2,3}$

A metodologia Lean, originária no Japão, tem sido aplicada em diferentes organizações, pois busca enxugar os desperdícios e possui foco na produtividade. Muitas empresas, incluindo os hospitais, estão adotando os princípios do modelo com objetivo de simplificar processos e eliminar atividades que não agregam valor. Os princípios Lean VSM Value Stream Maping e Kaisen permitem o mapeamento dos processos, a busca de gargalos e a definição de tarefas. Aidentificação dos processos chave é crucial e deve ser realizada juntamente com a equipe para entendimento de como a rotina realmente acontece e não como ela deveria ocorrer ${ }^{4-6}$.
Outra metodologia alinhada à melhoria de processos na área de aquisições é o Strategic Sourcing. Conceituado como um processo estruturado de compras, a ferramenta permite a identificação de oportunidades estratégicas através da análise sistemática da situação atual, incluindo as etapas de diagnóstico e análise de gastos, mapeamento da base de fornecedores, entendimento dos processos e competências, avaliação dos cenários externo e interno, bem como a implantação de processos colaborativos entre equipes internas multifuncionais e fornecedores ${ }^{7}$.

Por sua vez, a gestão de riscos, segundo Assi², permite uma avaliação dos efeitos internos e externos à empresa que podem impactar negativamente nas suas atividades, ou seja, é o mapeamento dos principais riscos, impactos e probabilidade de ocorrência. Desta forma, os gestores podem organizar ações de controle e de gestão visando a minimização dos impactos ou contingências. Para a gestão e avaliação dos riscos, o modelo adotado seguiu o preconizado pelo Manual de Gestão de Riscos do TCU que define como objeto da gestão de riscos qualquer processo de trabalho, atividade, projeto, iniciativa ou ação de plano institucional, assim como os recursos que dão suporte à realização dos objetivos da Organização ${ }^{8}$.

No trabalho apresentando a seguir, verificouse a implementação de práticas de governança corporativa aplicadas nas aquisições de um hospital terciário, no sul do Brasil, que alavancaram os processos de compra através do uso de ferramentas atuais de gestão, como a avaliação de riscos e compliance, strategic sourcing e o modelo Lean de melhoria. Considerando esses princípios e o cenário econômico atual, é necessário que as empresas sejam cada vez mais produtivas, com menores custos, principalmente na área pública, garantindo competitividade com o mercado privado. A busca da melhoria contínua, otimizando processos e tornando-os sustentáveis e transparentes deve ser a principal prioridade dos gestores públicos no Brasil. Neste sentido, a gestão das compras públicas é tema cada vez mais presente e importante dentro da Administração Pública pelo seu caráter complexo e estratégico. Assim, entender, discutir 
e aprimorar as compras públicas, para além de um simples procedimento administrativo e operacional é imperativo para as empresas públicas Brasileiras serem cada vez mais eficientes.

Neste contexto, avaliar o cenário da cadeia de aquisições através da aplicação das ferramentas descritas, busca o aperfeiçoamento dos processos resultando na melhora de indicadores, tais como: taxa de aproveitamentos dos pregões e tempo de tramitação dos processos de aquisição, imprimindo maior transparência ao processo licitatório.

\section{MÉTODOS}

Esse trabalho foi realizado no âmbito da Coordenadoria de Suprimentos de um hospital público terciário de grande porte do Sul do país. Para análise do cenário interno, buscou-se a identificação dos principais processos, ligados à cadeia de suprimentos do Hospital nas aquisições, que neste caso, tratou especificamente das modalidades de dispensa de licitação e pregão eletrônico ${ }^{3,9}$. Este mapeamento foi realizado com a aplicação do método Lean VSM Value Stream Mapping e Kaizen, que permitiu a identificação das principais etapas vinculadas, através de um mapa e identificação dos possíveis gargalos. A etapa de coleta de dados ocorreu através de busca ativa no sistema corporativo de gestão e informação eletrônica do hospital, mensurando o tempo dispendido nos processos licitatórios e a taxa de aproveitamento de pregões.

A ferramenta Strategic Sourcing foi utilizada para construção de modelos de negócio, com o mapeamento de novas possibilidades na cadeia de fornecimento. As equipes envolvidas, no fluxo de trabalho, foram alinhadas para prospectar produtos, condições comerciais e de entregas, fornecedores no mercado nacional, novas marcas e modelos.

$\mathrm{Na}$ sequência, os processos licitatórios da instituição foram classificados considerando os principais riscos envolvidos no certame e o impacto assistencial, conforme o Quadro 1, os quais foram definidos através de critérios de prioridade para a aquisição, podendo ser de baixa, média, alta ou imediata, conforme a proporção a seguir:

Quadro 1: Critérios de prioridade para as aquisições.

\begin{tabular}{|l|c|}
\hline \multicolumn{1}{|c|}{ Critério } & Peso \\
\hline Valor do contrato & $30 \%$ \\
\hline Itens fracassados anteriormente & $20 \%$ \\
\hline Licitação da agenda & $10 \%$ \\
\hline Impacto no paciente & $25 \%$ \\
\hline Impacto na atividade fim & $15 \%$ \\
\hline
\end{tabular}

Fonte: elaboração própria.
A partir da avaliação de cada critério, alinhado ao percentual atribuído de peso, foi concedida uma nota final ao processo de aquisição resultando na prioridade da contratação. Assim, é possível realizar o monitoramento de tempo de cada processo contemplando todas as suas etapas.

\section{RESULTADOS}

O trabalho realizado na gestão da cadeia de suprimentos do Hospital de Clínicas de Porto Alegre (HCPA), aplicando as ferramentas descritas trouxe uma série de importantes resultados que aprimoraram a governança das contratações, dando mais segurança e confiabilidade aos processos Institucionais. Na avaliação das ações desenvolvidas algumas puderam ser mensuradas e comparadas com os resultados de exercícios anteriores, como a Taxa de Aproveitamento dos Pregões, conforme apresentado na Tabela 1.

Tabela 1: Taxa de aproveitamentos dos pregões.

\begin{tabular}{cc}
\hline Ano & \% aproveitamento \\
2016 & $67 \%$ \\
2017 & $67 \%$ \\
2018 & $74,73 \%$ \\
2019 & $76,68 \%$ \\
2020 & $77,85 \%$ \\
\hline
\end{tabular}

Fonte: Sistema BASE HCPA (2020).

Através da tabela acima é possível verificar que desde 2016 vem ocorrendo melhora deste indicador, sendo que em 2020 tivemos o melhor resultado, o que representa maior assertividade no processo, não gerando retrabalho das equipes envolvidas em relação a republicação de lotes, reduzindo o tempo de ressuprimento e evitando lacunas de abastecimento.

$\mathrm{Na}$ Tabela 2 é possível analisar os resultados pelos grupos de materiais trabalhados no estudo. Comparando os resultados de 2018 e 2019 verifica-se uma importante melhora nos índices, onde o grupo de materiais de laboratório e diagnóstico destaca-se como melhor resultado.

Após aplicação dos conceitos da Gestão de Riscos, foi possível medir o indicador Tempo de Tramitação dos Processos. A priorização dos processos para as aquisições foi uma estratégia com impacto positivo, pois permitiu que processos cujos itens que apresentavam maior relevância ou impacto para a Instituição tivessem maior celeridade do que outros. Antes da implantação da estratégia, o tempo médio dos processos de compras classificados como pregão no sistema eletrônico de dados "SEI" em 2018 foi de 130 dias, em 2019 de 146 dias e em 2020 de 89 dias. Os resultados desse indicador são apresentados na Tabela 3. 
Pires et al.

Tabela 2: Resultados obtidos nas licitações por grupo de natureza e despesa.

\begin{tabular}{lccccc}
\hline \multicolumn{1}{c}{ Agenda } & $\begin{array}{c}\mathbf{N}^{\circ} \text { processos } \\
\text { homologados }\end{array}$ & $\mathbf{N}^{\circ}$ itens & $\begin{array}{c}\text { \% de } \\
\text { Aproveitamento } \\
\text { de lotes 2019 }\end{array}$ & $\begin{array}{c}\text { \% de } \\
\text { aproveitamento } \\
\text { de lotes 2018 }\end{array}$ & $\begin{array}{c}\text { Dif. pontos } \\
\text { percentuais }\end{array}$ \\
\hline Medicamentos & 27 & 649 & $70,05 \%$ & $64,22 \%$ & $5,83 \%$ \\
OPME & 23 & 669 & $74,56 \%$ & $69,12 \%$ & $5,44 \%$ \\
$\begin{array}{l}\text { Material Médico } \\
\text { Hospitalar }\end{array}$ & 32 & 688 & $86,48 \%$ & $78,49 \%$ & $7,99 \%$ \\
$\begin{array}{l}\text { Laboratório e } \\
\text { Diagnóstico }\end{array}$ & 7 & 128 & $87,86 \%$ & $71,97 \%$ & $15,89 \%$ \\
\hline
\end{tabular}

Fonte: Sistema IG.

Tabela 3: Tempo médio de processos - 2020.

\begin{tabular}{lcc}
\hline & $\begin{array}{c}\mathbf{N}^{\circ} \text { processos } \\
\text { homologados }\end{array}$ & $\begin{array}{c}\text { Tempo médio } \\
\text { de processo }\end{array}$ \\
\hline Imediato & 93 & 60 dias \\
Prioridade alta & 85 & 101 dias \\
Prioridade média & 65 & 101 dias \\
Prioridade baixa & 214 & 95 dias \\
\hline
\end{tabular}

Fonte: Sistema SEI (2020).

\section{DISCUSSÃO}

Conforme o Acórdão n 1.545/16, a governança das aquisições consiste no conjunto de mecanismos de liderança, estratégia e controle postos em prática para avaliar, direcionar e monitorar a atuação da gestão das aquisições, com o objetivo que as aquisições agreguem valor ao negócio da organização, com riscos aceitáveis ${ }^{10}$.

Segundo Fenili1" , a governança das aquisições tem por objetivo:

- Alinhar as políticas e as estratégias de gestão das aquisições às prioridades do negócio da organização em prol de resultados;

- Assegurar a utilização eficiente de recursos;

- Otimizar a disponibilidade e o desempenho dos objetos adquiridos;

- Mitigar riscos nas aquisições;

- Auxiliar a tomada de decisão sobre aquisições;

- Assegurar o cumprimento dos papéis e das responsabilidades, e a transparência dos resultados na função aquisição;

- Segregação de Funções;

- Transparência;

- Implementação da cultura de Gestão de Riscos e Compliance.
Com base no art. $2^{\circ}$, II, da Resolução-TCU 247/2011, pode-se entender governança das aquisições como o conjunto de diretrizes, estruturas organizacionais, processos e mecanismos de controle que visam assegurar que as decisões e as ações relativas à gestão das aquisições estejam alinhadas às necessidades da organização, contribuindo para o alcance das suas metas.

As etapas do processo licitatório e também dos dados coletados, foi possível identificar oportunidades de melhoria e otimização, principalmente nas licitações voltadas aos grupos de medicamentos, órteses e próteses (OPME), material médico Hospitalar e materiais de laboratório e diagnóstico que compreendem cerca de $75 \%$ do consumo da Instituição. Estes grupos de materiais possuem nichos específicos de mercado, que atendem as premissas do método Strategic sourcing.

Um ponto importante abordado na Governança das Contratações é a segregação de funções, que consiste na separação das atividades de autorização, aprovação, execução, controle e contabilização, conforme Silva ${ }^{12}$. Para evitar conflitos de interesses, é necessário repartir funções entre os servidores para que não exerçam atividades incompatíveis, como executar e fiscalizar uma mesma atividade. A segregação de funções destina-se a reduzir as oportunidades que permitam a qualquer pessoa estar em posição de perpetrar e de ocultar erros ou fraudes no curso normal das suas funções. Através da avaliação do mapeamento dos processos, optou-se por deslocar a equipe de formação de preços para a área de planejamento e alocar os pregoeiros por rodízio.

Além da busca por maior competitividade e assertividade nos processos entendeu-se a necessidade de avaliar e mensurar os riscos atribuídos aos objetos licitados. A gestão de riscos foi introduzida nas equipes através da elaboração de matrizes de riscos dos processos críticos de suprimentos e também na definição de prioridades de aquisição.

Para imprimir agilidade às compras na instituição, desenvolveu-se critérios de classificação para definição da prioridade a ser dada ao processo e atrelado 
a isso a criação de metas de tempo de processo. Neste sentido, foram criados critérios claros os quais foram atribuídas notas e pesos que combinados resultam na prioridade final do processo que pode ser classificado como imediato, alta prioridade, média prioridade ou baixa prioridade. Os critérios definidos foram:

- Valores financeiros dos lotes dos processos peso de $30 \%$;

- Processo resultante de um fracasso anterior peso de $20 \%$;

- A Licitação é da "agenda planejada" - peso de $10 \%$;

- Os produtos produzem impacto direto no paciente - peso de $20 \%$;

- Os produtos produzem impacto direto na atividade fim - peso de $20 \%$.

Essa classificação além de sinalizar a prioridade atribuída a cada processo gerou uma conexão entre as equipes que tramitam com os processos.

Através do uso das ferramentas descritas acima foi possível simularmos o volume de processos e os nichos de mercado atendidos pelo método Strategic Sourcing, bem como o impacto financeiro e as prioridades a serem definidas. É importante comentar que os grupos de despesas trabalhados neste método tratam das principais aquisições da Instituição. Desta forma, implementou-se as "agendas" de licitação, em que foi possível dimensionarmos, com maior precisão, o volume de trabalho e os resultados esperados. Estas agendas foram amplamente divulgadas através das mídias sociais do Hospital, evento com fornecedores, site e emails, contribuindo para a transparência das ações.

Conforme o Tribunal de Contas da União ${ }^{8}$, a transparência pública, além de possuir um papel fundamental no combate à corrupção, viabiliza a contribuição tempestiva da sociedade e dos órgãos de controle, no fornecimento de elementos para que o Estado se torne cada vez mais eficiente e efetivo.
Essa premissa é fundamental nas Instituições pois estimula o desenvolvimento de uma cultura de integridade na gestão dos negócios públicos e incentiva o esforço por melhores políticas e programas de governo.

\section{CONCLUSÕES}

O aprimoramento da governança nas contratações, principalmente das Instituições públicas, vem se tornando primordial e necessária para a manutenção da sustentabilidade econômica e transparência e confiabilidade.

As aquisições necessárias para o funcionamento de um Hospital de alta complexidade, que realiza assistência, ensino e pesquisa, voltados aos pacientes do Sistema Único de Saúde, convênios e privados são especialmente desafiadoras pela variabilidade de produtos, grupos, marcas e modelos. São inúmeras legislações a serem atendidas, desde licitações, contratos e de vigilância em saúde.

O uso de ferramentas como o Lean e o Strategic Sourcing permitiram a construção de modelos baseados em critérios que se mostraram bastante efetivos aplicados à realidade Institucional.

O uso do mapeamento dos principais riscos, baseados nas recomendações do Tribunal de Contas da União, tornou o processo mais seguro e transparente, permitindo a mitigação de riscos e a definição de critérios claros de prioridade nas aquisições, dando maior celeridade dos processos. O ganho obtido no grau de prioridade está em atender de forma mais rápida produtos e serviços que estão mais voltados à necessidade Institucional ou que repercutem de sobremaneira no orçamento.

A proposta de reorganização, baseada nos princípios da eficiência, transparência e compromisso, com os resultados e propósitos da instituição, foram os propulsores para imprimirmos um novo modelo de trabalho. A implementação de estratégias inovadoras acrescentam qualidade no trabalho e alto índice de satisfação dos agentes públicos.

\section{REFERÊNCIAS}

1. Brasil. Decreto $n^{\circ} 9.203$, de 22 de novembro de 2017: dispõe sobre a política de governança da administração pública federal direta, autárquica e fundacional. Diário Oficial da União [Internet]. 23 nov 2017 [citado em 25 fev 2020];1:3. Disponível em: http://www.planalto.gov.br/ccivil_03/ ato2015-2018/2017/decreto/D9203.htm
2. Assi M. Gestão de riscos com controles internos: ferramentas, certificações e métodos para garantir a eficiência dos negócios. São Paulo: Saint Paul; 2012.

3. Brasil. Lei $n^{0} 13.303$, de 30 de junho de 2016: dispõe sobre o estatuto jurídico da empresa pública, da sociedade de economia mista e de suas subsidiárias, no âmbito da União, dos Estados, do Distrito Federal e dos Municípios. Diário Oficial da União [Internet]. 1 jul 2016 [citado em 14 abr 2021];1:1. Disponível em: http://www. planalto.gov.br/ccivil_03/_ato20152018/2016/lei//13303.htm 
4. Martin K, Osterling M. Value stream mapping: how to visualize work and align leadership for organizational transformation. New York: McGrawHill Education; 2014.

5. Rodrigues VM. Entendendo, aprendendo e desenvolvendo: sistema de produção Lean Manufacturing. 2a ed. Rio de Janeiro: Campus; 2016.

6. Graban M. Lean hospitals: improving quality, patient safety, and employee engagement. 3rd ed. New York: Productivity Press; 2016.

7. Bim C. Strategic Sourcing: manual de aplicação da metodologia de compras estratégicas. 2a ed. São Paulo: Inllace; 2015.
8. Tribunal de Contas da União. Manual de gestão de riscos do TCU. Brasília, DF: TCU; 2018.

9. Brasil. Lei $n^{\circ} 10.520$, de 17 de julho de 2002: institui, no âmbito da União, Estados, Distrito Federal e Municípios, nos termos do art. 37, inciso XXI, da Constituição Federal, modalidade de licitação denominada pregão, para aquisição de bens e serviços comuns, e dá outras providências. Diário Oficial da União [Internet]. 18 ago 2002 [citado em 14 abr 2021];1:1. Disponível em: http://www.planalto.gov.br/ccivil_03/ leis/2002/l10520.htm

10. Brasil. Plenário Tribunal de Contas da União. Acórdão $n^{\circ}$ 1.545/16 [Internet]. Brasília, DF: TCU; 2016 [citado em 25 mar 2021].

Disponível em: https://portal. tcu.gov.br/data/files/1F/15/3F/8

D/8A285510BBC6B5551A281

8A8/023.246-2014- 6\%20ANOP\%20

UFC $\% 20$ governan_a $\% 20$ e $\% 20$

gest_o\%20de\%20aquisi es.pdf

11. Fenili R. Boas práticas administrativas em compras e contratações públicas. Niterói: Impetus; 2016.

12. Silva MA. O princípio da segregação de funções e sua aplicação no controle processual das despesas: uma abordagem analítica pela ótica das licitações públicas e das contratações administrativas. Revista do TCU. 2013;128:39-51.

Recebido: 30 jul, 2020 Aceito: 23 jun, 2021 